\title{
Effect of Leading-Edge Bulges on Aerodynamic Characteristics of Bionic Wingsail
}

\author{
Chen $\mathrm{LI}^{\mathrm{a}, \mathrm{b}, \mathrm{c}, 1}$, Peiting SUN ${ }^{\mathrm{a}}$ and Hongming WANG ${ }^{\mathrm{b}, \mathrm{c}}$ \\ a Marine Engineering College, Dalian Maritime University, Dalian, China \\ b Jiangsu Maritime Institute, 309 Gezhi Road, Jiangning District, Nanjing, China \\ c Jiangsu Marine Energy Saving and Emission Reduction Engineering Research and \\ Technology Development Center, Nanjing, China
}

\begin{abstract}
The leading-edge bulges along the extension direction are designed on the marine wingsail. The height and the spanwise wavelength of the protuberances are $0.1 c$ and $0.25 c$, respectively. At Reynolds number $R e=5 \times 10^{5}$, the Reynolds Averaged Navier-Stokes equations are applied to the simulation of the wingsail with the bulges thanks to ANSYS Fluent finite-volume solver based on the SST K- $\omega$ models. The grid independence analysis is carried out with the lift and drag coefficients of the wingsail at $\mathrm{AOA}=8^{\circ}$ and $\mathrm{AOA}=20^{\circ}$. The results show that while the efficiency of the wingsail is reduced by devising the leading-edge bulges before stall, the bulges help to improve the lift coefficient of the wingsail when stalling. At $\mathrm{AOA}=22^{\circ}$ under the action of the leading-edge tubercles, a convective vortex is formed on the suction surface of the modified wingsail, which reduces the flow loss. So the bulges of the wingsail can delay the stall.
\end{abstract}

Keywords. Leading-edge bulges, aerodynamic characteristics, bionic wingsail

\section{Introduction}

Because of its characteristics of environmental protection, wingsail began to be applied in various types of auxiliary propulsion devices with ships. In 1980, Japan installed two wingsails on the "Shin Aitoku Maru". After four years of actual navigation, the ship saved $8.5 \%$ of fuel oil per year than the conventional ship [1]. Because of the remarkable energy saving effect of the wingsail, the research on sail-assisted ships varies from country to country. However, when the angle of attack is too large, the suction surface of the wingsail will have airflow separation, or even stall, and the propulsion performance of the wingsail will deteriorate. In the 35th America's cup, the New Zealand chiefs suffered a capsizing accident [2]. So, it is necessary to find a way to control the separation or delay the stall. Fish [3] found in the cross-section of the humpback fins that the rounded bulges were regularly interspersed on the leading edge. The humpback whale maintains lift at high angles of attack by controlling flow over the flipper. Zhang et al. [4] studied the influence of bulges on performance of wing and the mechanism of induced flowing. They all believed that the working mechanism of protuberances is similar to that of traditional vortex generator. Johari [5] proposed that the presence of

${ }^{1}$ Corresponding Author, Chen LI, Marine Engineering College, Dalian Maritime University, Dalian, China; E-mail: lichen0987654321@163.com. 
protuberances is not conducive to the pre-stall performance of the wing, but beneficial to the performance after stall. The bulges are identified as post stall, resulting in enhanced momentum transfer effect, reduced flow separation and increased lift. Gao [6] studied the influence of rudder with the bulges on hydrodynamic characteristics.

Based on the above research, a modified wingsail with leading-edge tubercles is designed to improve the aerodynamic performance of the sail. NACA 0018 wing is used as the baseline airfoil of the rectangular sail, and the effect of protuberances on the propulsion performance of the wingsail is numerically studied. Furthermore, the stall mechanism and aerodynamic characteristics of the wingsail with leading-edge protuberance is revealed.

\section{Numerical Models and Methods}

\subsection{Design Scheme of Protuberances of Wingsail}

The wingsail with tubercles consist of some sections with different chord lengths of NACA0018. The spanwise wavelength $(\lambda)$ of the leading-edge tubercles were simulated, which was assumed $0.25 \mathrm{c}$ and the amplitude $(A)$ is set as $0.1 \mathrm{c}$. According to those designed parameters, the $3 \mathrm{D}$ models of initial and modified wingsails are showed in figure 1 .

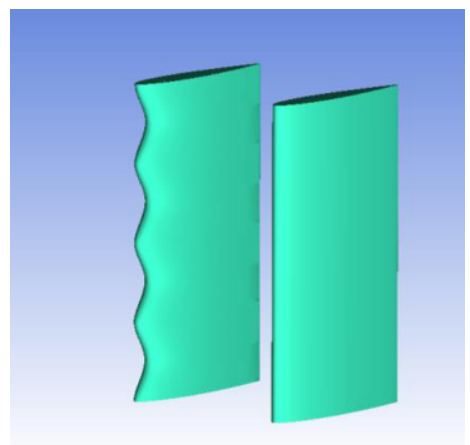

Figure 1. Modified wingsail and initial wingsail.

\subsection{Computational Domain of the Wingsail}

The computational domain of the wingsail is a cuboid as figure 2 , which is $30 \mathrm{c}$ in length, $20 \mathrm{c}$ in width and 10c in height. The inlet is located at $8 \mathrm{c}$ upstream from the wingsail. In order to ensure that the bottom boundary has no effect on the flow field of the wingsail, the distance between the bottom of the wingsail model and that of the computational domain is set as $4 \mathrm{c}$. The chord of the wing is $0.35 \mathrm{~m}$ and the chord ratio is 2 . The atmospheric boundary layer is not considered to simplify the model. The air inlet surface is simulated on five sides (inlet boundary, starboard boundary, port boundary, top boundary, bottom boundary) and the velocity is uniform as the free flow velocity. The outlet pressure is set to atmospheric pressure. The surfaces of wingsail are set as non-slip wall. 


\subsection{Mesh Details}

The accuracy of numerical results is determined by the quality of mesh. The grid quantity and quality of the computational domain are well set, and the surface grid of the sail is shown in figure 3. In addition, the grid size of the tubercles of the sail is set as $0.4545 \% \mathrm{c}$ with 9.86 million grids. In order to accurately simulate the flow field near the wall, the $\mathrm{y}+$ of the sail with $\mathrm{AOA}=8^{\circ}$ is about 1 , where $\mathrm{y}$ is specified as $2.871 \times 10^{-5} c$.

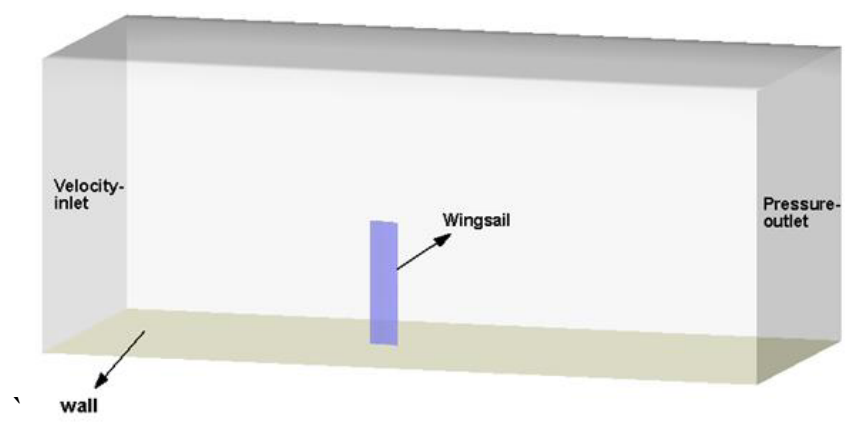

Figure 2. The computational domain.

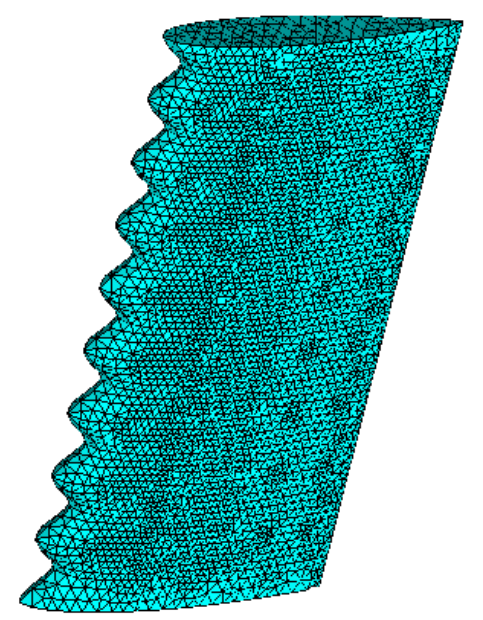

Figure 3. Surface meshes of the modified wingsail.

\subsection{Mesh Independence}

It is very important to analyze the effect of mesh numbers. When $R e=5 \times 10^{5}$, different mesh numbers are used to estimate grid sensitivity with 2.5 million, 4.22million, 9.86million and 14.4 million mesh numbers. The grid independence analysis is carried out with the lift and drag coefficients of the wingsail at $\mathrm{AOA}=8^{\circ}$ and $\mathrm{AOA}=20^{\circ}$ as shown in figure 4 . According to these calculations, the error of lift coefficient is less than $0.3 \%$ between 9.86 million and 14.4 million while there is almost no error in the drag coefficient. And the increase of grid number leads to a small change in lift, which can be considered as acceptable. Therefore, the mesh number of 9.86 million is adopted in the paper. 


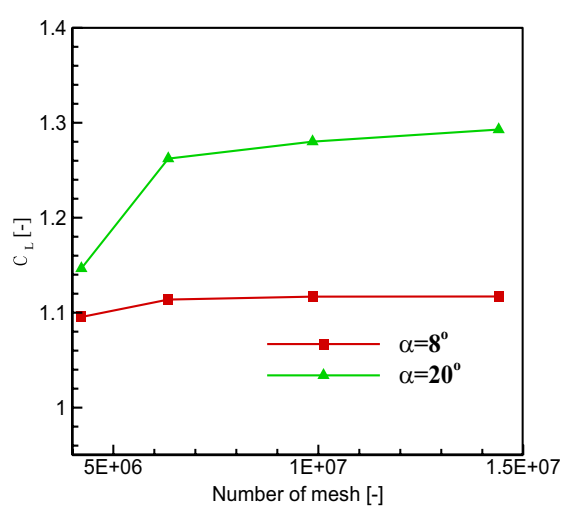

(a) $C_{\mathrm{L}}$ vs mesh number

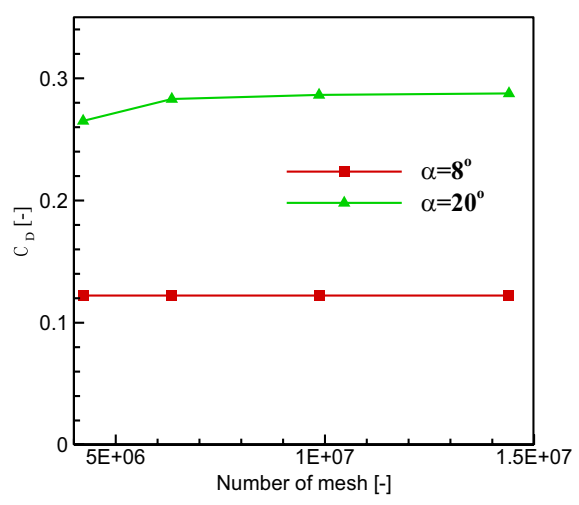

(b) $C_{\mathrm{D}}$ vs mesh number

Figure 4. Convergence of $\alpha=8^{\circ}$ and $\alpha=20^{\circ}$ as a function of mesh number.

\subsection{Computational Approach}

The Reynolds Averaged Navier-Stokes equations are applied to the simulation thanks to ANSYS Fluent finite-volume solver based on the SST K- $\omega$ models. Because of the low Reynolds number $\left(R e=5 \times 10^{5}\right)$, the air is considered as incompressible. The turbulence intensity at inlet is $3 \%$. The QUICK scheme is used for the discretization scheme, and the discrete format is quick. Simple algorithm is adopted for the pressure velocity coupling scheme [7].

\section{Calculation Results}

The lift coefficient $C_{L}$ and drag coefficient $C_{D} \mathrm{~s}$ are two aerodynamic performances of the sail. They are expressed as follows [8]:

$$
\begin{gathered}
C_{L}=\frac{L}{0.5 \rho V_{R}^{2} A_{R}} \\
C_{D}=\frac{D}{0.5 \rho V_{R}^{2} A_{R}}
\end{gathered}
$$

where $L$ is the lift and $D$ is drag force of the wingsail.

\subsection{Model Validation}

The experimental results are compared with the numerical results to validate numerical simulation, as shown in figure 5. The experimental results come from Daniel's doctoral dissertation, which is an experiment on the aerodynamic characteristics of NACA 0018 [9]. For the case of $R e=3.2 \times 10^{6}$, The validation of the Reynolds Averaged Navier-Stokes equations and the unsteady Reynolds Averaged Navier-Stokes equations method is used. The results of the drag coefficient and lift coefficient are close to the experimental values before stall $\left(\mathrm{AOA}<22^{\circ}\right.$ ), and the estimation error is less than $4 \%$ between the numerical 
results and the test values after stall. Therefore, the numerical method is suitable for simulating the wingsail.

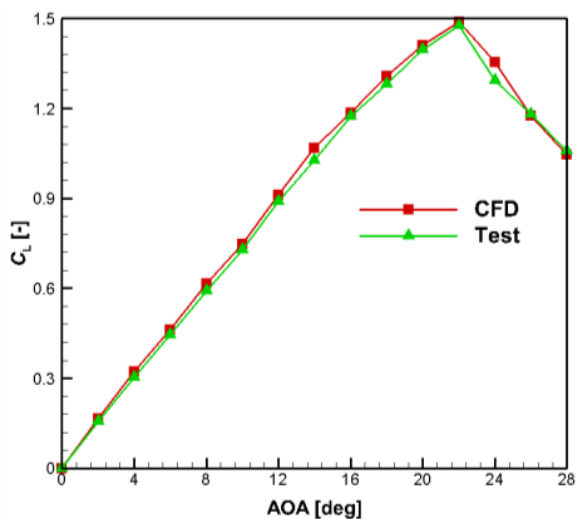

(a) Lift coefficient $C_{L}$ vs AOA

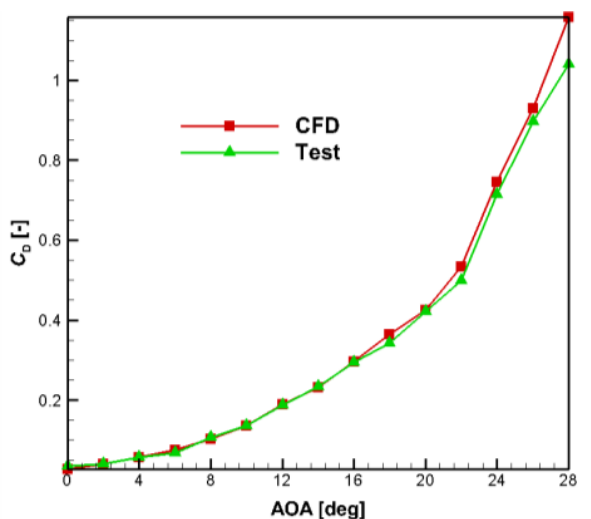

(b) Drag coefficient $C_{D}$ vs AOA

Figure 5. Comparison between experiment [9] and numerical simulation.

\subsection{Aerodynamic Performance}

It can be seen from figure 6 that when the angle of attack increases, the lift coefficient of unmodified wingsail increases linearly from $0^{\circ}$ to $16^{\circ}$ and the drag coefficient is low, about 0.1 . The lift coefficient increases gently from $16^{\circ}$ to $20^{\circ}$ and maintains at about 0.85 , while the drag coefficient increases slightly from 0.1 to about 0.14 . After the angle of attack exceeds $21^{\circ}$, the unmodified wingsail enters the deep stall state, the lift coefficient suddenly drops to about 0.4 , and the drag coefficient suddenly increases from 0.14 to about 0.33 . After the angle of attack exceeds $22^{\circ}$, the lift coefficient keeps at about 0.4 , decreases slowly. At $30^{\circ}$ the drag coefficient is close to the lift coefficient of unmodified wingsail.

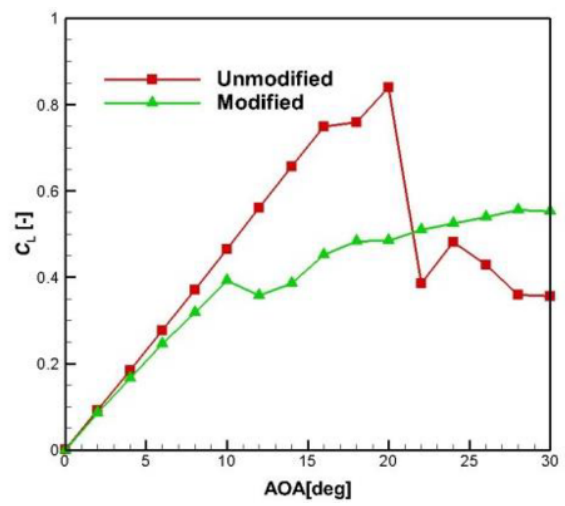

(a) Lift coefficient vs AOA

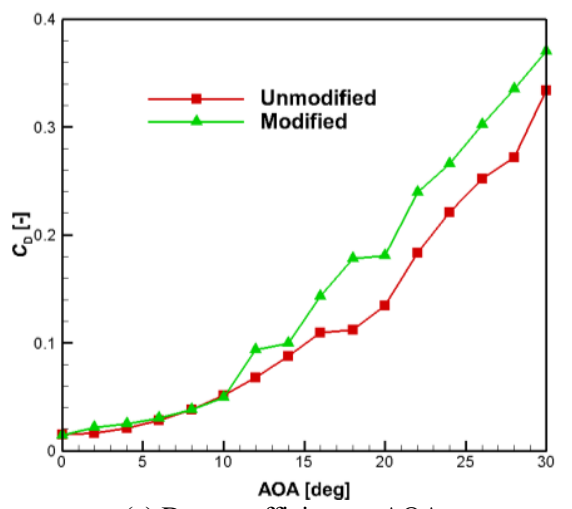

(a) Drag coefficient vs AOA

Figure 6. Lift coefficient and drag coefficient.

For the modified wingsail, the lift coefficient in the linear growth region is slightly lower than that of the unmodified wingsail when the angle of attack increases. And the lift coefficient reaches the maximum at about $10^{\circ}$. The lift coefficient decreases slightly 
from $10^{\circ}$ to $14^{\circ}$ and the drag coefficient increases obviously, which is higher than that of unmodified wingsail. The lift coefficient is maintained at 0.5-0.6 when the angle of attack is $16^{\circ}-30^{\circ}$. Then the lift coefficient is higher than that of unmodified wingsail after the angle of attack exceeds $22^{\circ}$. At the same time, the drag coefficient of modified wingsail is close to that of unmodified wingsail.

\subsection{Streamlines}

As for the unmodified wingsail and modified wingsail at $\mathrm{AOA}=12^{\circ}$, the streamlines on the $\mathrm{z} / c=0.5$ plane are depicted in figure 7 . It can be seen that while the lift coefficient decreases slightly from $10^{\circ}$ to $14^{\circ}$, there is no separation vortex appears over the suction surface of the modified wingsail because of the bulges. However, the lift coefficient of the initial wingsail is lower than that of the modified wingsail at $\mathrm{AOA}=22^{\circ}$. The streamlines on the $\mathrm{z} / c=0.5$ plane at $\mathrm{AOA}=22^{\circ}$ are depicted in figure 8 .

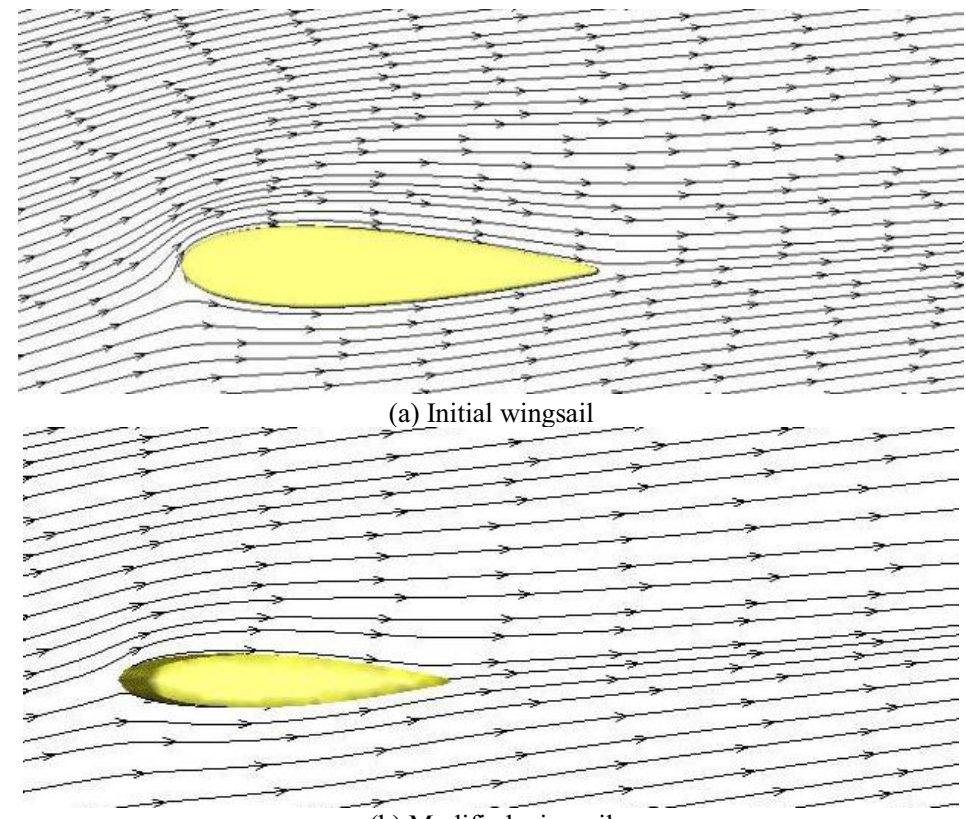

(b) Modified wingsail

Figure 7. Streamlines at $\mathrm{AOA}=12^{\circ}(\mathrm{z} / \mathrm{c}=0.5$ plane $)$.

It can be seen that the vortex of the modified wingsail is smaller than the unmodified wingsail. Under the action of the bulges, a convective vortex is formed on the suction surface of the modified wingsail, which reduces the flow loss. It helps explain that the lift coefficient of the modified wingsail is larger than that of the initial wingsail at $A O A=$ $22^{\circ}$. 


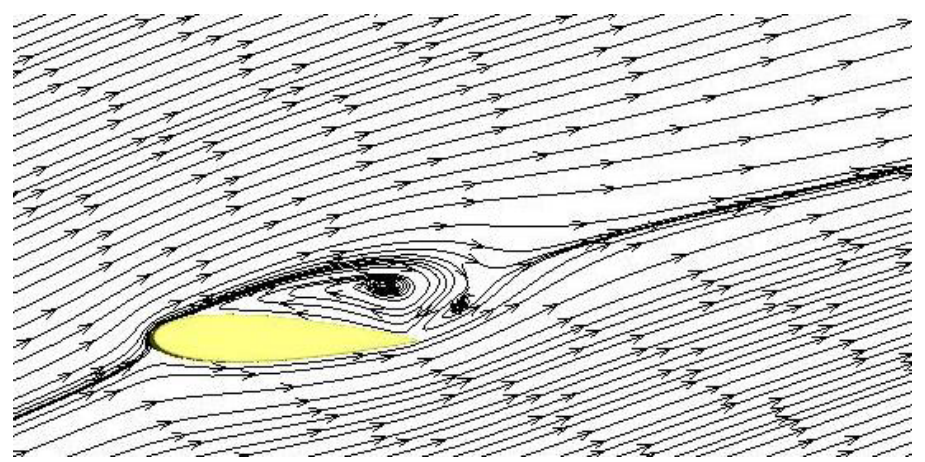

(a) Initial wingsail

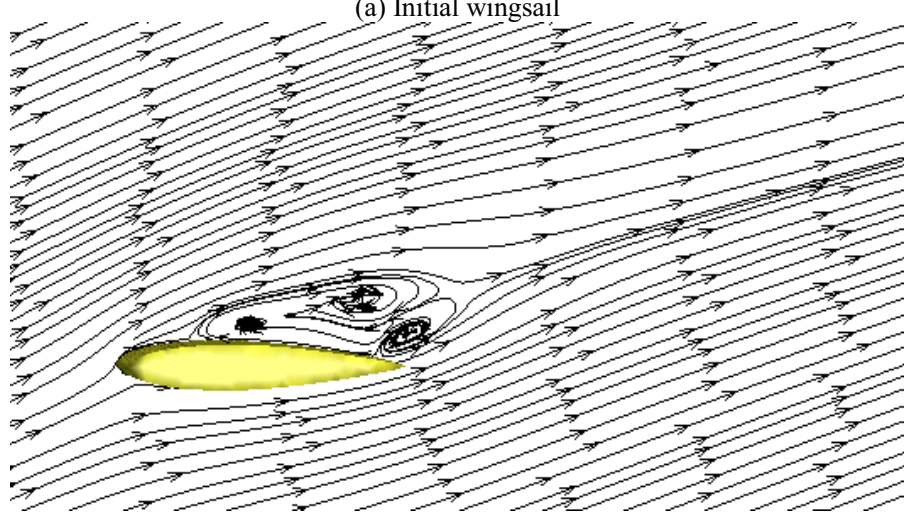

(b) Modified wingsail

Figure 8. Streamlines at $\mathrm{AOA}=22^{\circ}(\mathrm{z} / c=0.5$ plane $)$.

\section{Conclusion}

In this article, the effects of the pulges are analyzed through simulations. The height and the spanwise wavelength of the protuberances are $0.1 \mathrm{c}$ and $0.25 \mathrm{c}$, respectively.

For the modified wingsail, the lift coefficient in the linear growth region is slightly lower than that of the initial wingsail when the angle of attack increases from $0^{\circ}$ to $16^{\circ}$. The lift coefficient is maintained at $0.5-0.6$ when the angle of attack is $16^{\circ}-30^{\circ}$. Then the lift coefficient is higher than that of initial wingsail after the angle of attack exceeds $22^{\circ}$. For the initial wingsail, the lift coefficient increases gently from $16^{\circ}$ to $20^{\circ}$ and maintains at about 0.85 . After the angle of attack exceeds $22^{\circ}$, the unmodified wingsail enters the deep stall state, which is not conducive to the safe operation of the wingsail.

While the lift coefficient decreases slightly from $10^{\circ}$ to $14^{\circ}$, there is no separation vortex appears over the suction surface of the modified wingsail because of the protuberances. At $\mathrm{AOA}=22^{\circ}$ under the action of the bulges, a convective vortex is formed on the suction surface of the modified wingsail, which reduces the flow loss. So the lift coefficient of the modified wingsail is larger than that of the initial wingsail at $\mathrm{AOA}=22^{\circ}$. 


\section{Acknowledgments}

This work is financially supported by Natural Science Research Projects in Jiangsu Higher Education institutions (20KJB580010, 20KJB580008), Research Funds of Jiangsu Maritime Institute (014070, kjcx-1907) from China.

\section{References}

[1] Ishihara M, Watanabe T, Shimizu K, et al. 1980 Prospect of sailequipped motor ship as assessed from experimental ship Daioh Proceedings of the Shipboard Energy Conservation Symposium (Society of Naval Architects and Marine Engineers) pp 181-198.

[2] Blakeley A W, Flay R G J and Richards P J 2012 Design and optimisation of multi-element wing sails for multihull yachts 18th Australasian Fluid Mechanics Conference (Australia).

[3] Fish F E and Lauder G V 2006 Passive and active flow control by swimming fishes and mammals Annu. Rev. Fluid Mech. 38 193-224.

[4] Zhang M M, Wang G F and Xu J Z 2013 Aerodynamic control of low-Reynolds-number airfoil with leading-edge protuberances AIAA J 51 (8) 1960-1971.

[5] Johari H, Henoch C, Custodio D, et al. 2007 Effects of leading-edge protuberances on airfoil performance AIAA J 45 (11) 2634-2642.

[6] Gao H and Zhu W 2020 Numerical investigation of bionic rudder with leading-edge protuberances $J$. Offshore Mech. Arct. Eng. 142 (1) 011802.

[7] Li C, Wang H M and Sun P T 2020 Numerical investigation of a two-element wingsail for ship auxiliary propulsion J. Mar. Sci. Eng. 8 333. doi:10.3390/jmse8050333

[8] Zhu W C and Gao H T 2018 Design and numerical investigation of rudder with leading-edge protuberances International Conference on Design Theory and Methodology.

[9] Daniel W A 1996 The CFD Assisted Design and Experimental Testing of a Wingsail with High Lift Devices (University of Salford: Salford, England). 